\section{Dr. Zhao, et al, reply}

\section{To the Editor:}

We thank Dr. Johannes Roth for his comments ${ }^{1}$ on our recent publication ${ }^{2}$. Dr. Roth raises several important points. The first is that for a Doppler signal to be considered abnormal it must exist within thickened synovium. This is based on adult studies and a recent consensus conference defining ultrasound (US) requirements for the pediatric population ${ }^{3}$. Physiologic blood flow may exist within the joint space of children without abnormal structural changes of the synovium. Thus, if these new definitions of Doppler signal detection in synovitis for children are applied to the images for our study, the presence of synovitis would have been much less frequent in our cohort. Thus, Roth argues that our conclusion that nearly half of children with clinically inactive disease had abnormal US findings would not be supported and that the poor predictive value of the baseline US in this patient population would be in question.

We agree that the data from adult studies is quite clear that Doppler signal within abnormal synovium on B-mode ultrasound correlates with histological findings of inflammation ${ }^{4}$. Based on these data, pediatric US interpretation was established and a consensus for interpretation was recently developed ${ }^{3}$. Dr. Roth also points out that many clinicians observed increased blood flow surrounding synovium in joints even with the resolution of structural synovial changes. The prevalence and significance of Doppler signal within the joint space in healthy children and children with inactive juvenile idiopathic arthritis (JIA) need to be investigated in a longitudinal study to understand the variation of Doppler signal within the same host over time and the predictive value of such signals in children with JIA without detectable expanded synovium. Our current data suggest that such signal did not predict future arthritis flares within the same joint. The presence of effusion and synovial thickening were very low for the wrists, knees, and tibiotalar joints in children with inactive JIA, and therefore the sensitivity of US to predict future flares was also low. We were not able to investigate the effect of continuing treatment on the presence or absence of Doppler signals (with or without expanded synovium detected by US). Interestingly, a recent study by De Lucia, et $a l^{5}$ also reported low sensitivity but better predictive value of flares when stringent criteria were applied and 44 joints were scanned.

In this evolving field, we agree with Dr. Roth that the definition of abnormal Doppler needs further standardization and investigation. Based on our limited sample size, even after we removed the data of Doppler signals without synovial thickening, clinical examination has shown excellent accuracy, which suggests that US may not be needed to determine disease status in children with clinically inactive JIA. However, a larger study with comprehensive joint scanning is needed to validate this hypothesis.

The other point Dr. Roth raises concerns the number of views used in our US assessments, specifically for the subtalar joint. We agree that multiple views of each joint provide more robust assessment and should be encouraged for future studies. At the time of our study in 2013, a single view of each joint was regarded as standard and thus was adopted for our study design.

We appreciate the opportunity to respond to the insightful comments from Dr. Roth ${ }^{1}$.

YONGDONG ZHAO, MD, PhD, Pediatric Rheumatology, Seattle Children's Hospital, Department of Pediatrics, University of Washington, and Center for Clinical and Translational Research, Seattle Children's Hospital; NANCI E. RASCOFF, MD, MPH, Pediatric Rheumatology, Seattle Children's Hospital, Department of Pediatrics, University of Washington; RAMESH S. IYER, MD, Pediatric Radiology, Seattle Children's Hospital, University of Washington; MAHESH THAPA, MD, Pediatric Radiology, Seattle Children's Hospital, University of Washington; CAROL A. WALLACE, MD, Pediatric Rheumatology, Seattle Children's Hospital, Department of Pediatrics, University of Washington, Seattle, Washington, USA. Address correspondence to Dr. Y. Zhao, MA 7.110, 4800 Sand Point Way NE, Seattle, Washington 98105, USA.

E-mail: yongdong.zhao@seattlechildrens.org

\section{REFERENCES}

1. Roth J. Predictive value of musculoskeletal ultrasound for flares in juvenile idiopathic arthritis. J Rheumatol 2019;46:113.

2. Zhao Y, Rascoff NE, Iyer RS, Thapa M, Reichley L, Oron AP, et al. Flares of disease in children with clinically inactive juvenile idiopathic arthritis were not correlated with ultrasound findings. J Rheumatol 2018;45:851-7.

3. Roth J, Jousse-Joulin S, Magni-Manzoni S, Rodriguez A, Tzaribachev N, Iagnocco A, et al. Definitions for the sonographic features of joints in healthy children. Arthritis Care Res 2015;67:136-42.

4. Koski JM, Saarakkala S, Helle M, Hakulinen U, Heikkinen JO, Hermunen H. Power Doppler ultrasonography and synovitis: Correlating ultrasound imaging with histopathological findings and evaluating the performance of ultrasound equipments. Ann Rheum Dis 2006;65:1590-5.

5. De Lucia O, Ravagnani V, Pregnolato F, Hila A, Pontikaki I, Gattinara M, et al. Baseline ultrasound examination as possible predictor of relapse in patients affected by juvenile idiopathic arthritis (JIA). Ann Rheum Dis 2018;77:1426-31.

J Rheumatol 2019;46:1; doi:10.3899/jrheum.180843 